\title{
Analysis of the anthropometric data of adults aged $65+$ years participating in the National Adult Nutrition Survey
}

\author{
E. A. Boylan ${ }^{1}$, B. A. McNulty ${ }^{1}$, J. Walton ${ }^{2}$, A. Flynn ${ }^{2}$, A. P. Nugent ${ }^{1}$ and M. J. Gibney ${ }^{1}$ \\ ${ }^{1}$ UCD Institute of Food and Health, University College Dublin, Belfield, Dublin 4, Ireland and ${ }^{2}$ School of Food and \\ Nutritional Sciences, University College Cork, Cork, Ireland
}

Understanding body composition, how it changes with age and any associated health implications is important to the health care and nutritional support of older populations ${ }^{(1)}$. The aim of this research was to obtain measured anthropometric data for those adults aged $65+$ years who participated in the National Adult Nutrition Survey (NANS) ${ }^{(2)}$.

Anthropometric measurements were performed by trained fieldworkers on consenting participants aged $65+$ years. Height was assessed using a Leicester portable height measure (Chasmores Ltd, UK) and waist to hip ratio calculated using tape measurements of waist and hip circumference. Weight and body composition were determined using a Tanita ${ }^{\circledR}$ weighing scale that is based on bio-electrical impedance analysis $^{(3)}$.

\begin{tabular}{|c|c|c|c|c|c|c|c|c|c|}
\hline & \multicolumn{3}{|c|}{ 65-69 years } & \multicolumn{3}{|c|}{$70-74$ years } & \multicolumn{3}{|c|}{$75+$ years } \\
\hline & $n$ & Mean & SD & $n$ & Mean & SD & $n$ & Mean & SD \\
\hline Weight (kg) & 68 & 74.3 & 11.5 & 61 & 77.9 & 16.7 & 59 & 71.5 & 12.5 \\
\hline Height (m) & 68 & 1.64 & 0.09 & 62 & 1.65 & 0.10 & 59 & 1.62 & 0.09 \\
\hline WHR & 61 & 0.90 & 0.09 & 54 & 0.92 & 0.08 & 55 & 0.91 & 0.08 \\
\hline BMI $\left(\mathrm{kg} / \mathrm{m}^{2}\right)$ & 68 & 27.4 & 3.4 & 61 & 28.5 & 4.8 & 59 & 27.1 & 4.1 \\
\hline Body fat (\%) & 65 & 32.4 & 7.6 & 53 & 33.4 & 7.3 & 45 & 32.6 & 7.6 \\
\hline FFM (kg) & 65 & 50.3 & 9.1 & 53 & 52.6 & 11.2 & 45 & 47.9 & 9.0 \\
\hline MM (kg) & 65 & 47.8 & 8.6 & 53 & 50.0 & 10.7 & 45 & 45.5 & 8.5 \\
\hline TBW (kg) & 65 & 34.8 & 6.3 & 51 & 36.4 & 8.4 & 45 & 32.9 & 6.7 \\
\hline
\end{tabular}

$n$, sample size; WHR, waist to hip ratio; \%: percentage; FFM, fat-free mass; MM, muscle mass; TBW, total body water.

There were no statistical differences between the three age groups (one-way ANOVA).

The table shows the mean and SD of anthropometric measurements across three age groups $(65-69,70-74$ and $75+$ years) for this older cohort of NANS. While no statistically significant differences (one-way ANOVA) in measurements were identified between the groups, it can be observed that those aged 70-74 years tended to have the highest mean values for all measurements. Comparison with NANS participants under 65 years of age highlighted a significantly lower weight and higher WHR compared with those participants over the age of 75 years $(P<0.05$, one-way ANOVA, with Tamhane and Scheffe post hoc tests, respectively). There were also significant differences $(P<0.05$, one-way ANOVA, with Tamhane post hoc test $)$ in percentage body fat; those in the younger cohort having a lower percentage body fat than each of the age groups in this cohort of adults aged $65+$ years.

This preliminary analysis reveals no major differences in body composition between the three age groups of older adults, however, differences in composition were observed when compared with the younger adults. Further analysis of this dataset will allow for more complete understanding of body composition in older Irish adults.

This study was funded by the Irish Department of Agriculture, Fisheries and Food under the Food for Health Research Initiative (2007-2012).

1. Chumlea CW \& Baumgartner RN (1989) Am J Clin Nutr 50, 1158-1166.

2. Irish Universities Nutrition Alliance (2011) The National Adult Nutrition Survey. http://www.iuna.net

3. Lukaski H, Johnson P et al. (1985) Am J Clin Nutr 41, 810-817. 